\title{
Regularizational approach for modeling ductile damage
}

\author{
Kai Langenfeld ${ }^{1}$, Kerstin Möhring ${ }^{2}$, Frank Walther ${ }^{2}$, and Jörn Mosler ${ }^{1, *}$ \\ ${ }^{1}$ Institute of Mechanics, TU Dortmund University, D-44227 Dortmund, Germany \\ ${ }^{2}$ Department of Materials Test Engineering, TU Dortmund University, D-44227 Dortmund, Germany
}

\begin{abstract}
It is well known, that modeling of material softening behavior can lead to ill-posed boundary value problems. This, in turn, leads to meshdependent results as far as the finite-element-method is concerned [1]. Several solution strategies in order to regularize the aforementioned problem have been proposed in the literature, cf. [2]. However, these strategies often involve high implementational effort. An approach which is very efficient from an implementational point of view is the so-called micromorphic approach by [3, 4]. This regularization technique includes gradients of internal variables implicitly into the framework, while preserving the original structure of the underlying local constitutive model. However, it is shown that a straightforward implementation of the micromorphic approach does not work for single-surface ductile damage models. By analyzing the respective equations, a modification of the micromorphic approach is proposed - first for a scalar internal variable, i.e., isotropic damage. Subsequently, the novel regularization method is extended to tensorvalued damage, i.e., anisotropic material degradation.
\end{abstract}

\section{Introduction}

Defects like pores or micro-cracks can be found in any technologically relevant material, cf. Fig. 1. Due to loading, additional defects might nucleate, existing defects might coalesce
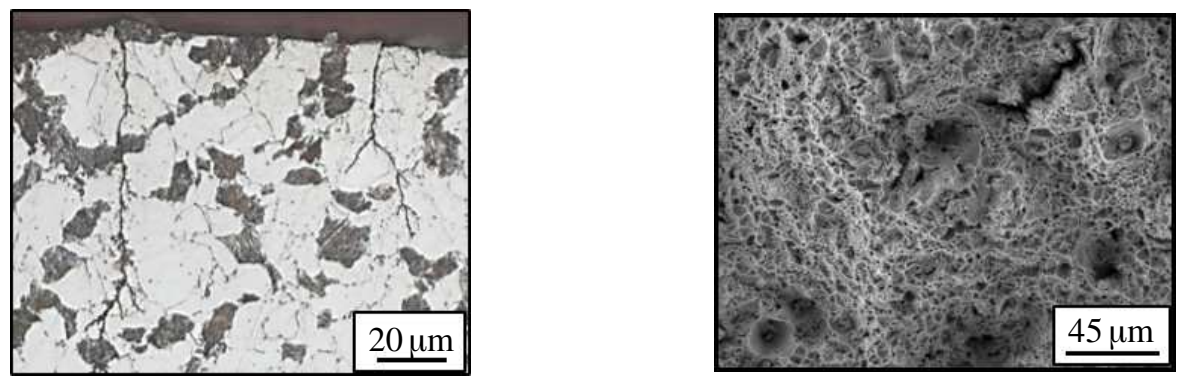

Figure 1. Defects in metals (taken from: SFB/TR TRR 188, project C01) - left: Micro cracks in a dual-phase steel; right: Accumulation of pores in 16MnCrS5

or simply evolve in size and shape. In order to describe the effect of such defects as well

\footnotetext{
*e-mail: joern.mosler@tu-dortmund.de
} 
as their evolution on the overall macroscopic response, continuum damage mechanics have been developed during the last decades, cf. [5, 6]. Although the macroscopic modeling of anisotropic damage accumulation is indeed challenging, nowadays several promising models/frameworks are available, see [7, 8]. They are sound with respect to fundamental physics principles such as the second law of thermodynamics and also with respect to certain invariance principles necessary within a geometrically exact setting, cf. [9].

As far as local models are concerned, continuum damage mechanics can be considered as a well established framework. However, these local models which do not involve any length scale are ill-posed from a mathematical point of view. This ill-posedness results in the well known pathological mesh dependence as far as their finite element implementations are concerned, cf. [1].

Two popular types of regularization techniques are non-local models and gradientenhanced models. Implementational aspects favor gradient-enhanced models, since no special additional data structure is required such as that for evaluating the non-local integrals, see [2]. An elegant framework for gradient-enhancement is the micromorphic approach by $[3,4]$. It implicitly incorporates gradients into the local constitutive model while preserving its original structure. To be more precise, inequalities due to loading/unloading conditions, which occur in classic gradient-enhanced models at the global finite element level can be restricted to the involved material/integration points.

Although promising local models for anisotropic damage and general regularization techniques exists in literature, the combination is rare. A few exceptions are $[10,11]$. The derivation of a general framework for gradient-regularized anisotropic damage models is precisely the goal of this paper. Although the framework to be developed is very general, the focus lies on ductile damage. For this reason, the model should rely on only one single failure/yield surface - in contrast to previous works.

The contribution is structured as follows: Section 2 briefly addresses the fundamentals of constitutive modeling. In Section 3 a general local framework suitable for modeling anisotropic material degradation is reviewed. This local model will be regularized in Section 4 by employing the micromorphic approach. Since a straightforward incorporation of the micromorphic ansatz does not work, a novel micromorphic approach is elaborated. The simplifications of the final anisotropic model for an isotropic damage evolution are derived in Section 5. Finally, the predictive capabilities of the fully anisotropic and the isotropic ductile damage model are analyzed in Section 6.

\section{Fundamentals}

Within this contribution, a geometrically linearized setting is adopted. This is motivated by the fact that the final model shall be used for low cycle fatigue analyses.

Let $\boldsymbol{u}(\boldsymbol{X})$ be the displacement field of all points $\boldsymbol{X}$ belonging to body $\mathcal{B}$ (the reference configuration). Then, total strain tensor $\varepsilon$ is defined as

$$
\boldsymbol{\varepsilon}=\frac{1}{2}\left[\nabla \boldsymbol{u}+\nabla^{T} \boldsymbol{u}\right]
$$

where $\nabla$ is the gradient operator with respect to $\boldsymbol{X}$. For modeling plasticity, total strain tensor $\varepsilon$ is additively decomposed into an elastic and a plastic part, i.e.,

$$
\varepsilon=\varepsilon^{\mathrm{e}}+\varepsilon^{\mathrm{p}}
$$

Focusing on statics, balance of linear momentum is given in local format by partial differential equation

$$
\operatorname{div} \sigma=-\rho_{0} \boldsymbol{B}_{0} \quad \forall \boldsymbol{X} \text { in } \mathcal{B}
$$


Here, div denotes the divergence operator, $\boldsymbol{\sigma}$ is the stress tensor and $\rho_{0} \boldsymbol{B}_{0}$ volume forces. By decomposing the boundary of body $\mathcal{B}$ into disjunct parts $\partial \mathcal{B}_{D}$ (Dirichlet boundary) and $\partial \mathcal{B}_{N}$ (Neumann boundary), the boundary conditions associated with partial differential equation (3) can be written as

$$
\boldsymbol{u}=\boldsymbol{u}^{*} \quad \forall \boldsymbol{X} \text { in } \mathcal{B}_{D}, \quad \boldsymbol{\sigma} \cdot \boldsymbol{N}=\boldsymbol{T}^{*} \quad \forall \boldsymbol{X} \text { at } \partial \mathcal{B}_{N},
$$

in which $\boldsymbol{N}$ is the normal vector at boundary $\partial \mathcal{B}$, and in which $\boldsymbol{T}^{*}$ are prescribed tractions acting at $\partial \boldsymbol{B}_{N}$.

\section{Anisotropic ductile damage - Constitutive Framework}

A simplified version of the Helmholtz energy for the anisotropic ductile damage model proposed in [7] is given as

$$
\Psi=\frac{\Lambda}{2} \operatorname{tr}\left(\boldsymbol{b} \cdot \boldsymbol{\varepsilon}^{\mathrm{e}}\right)^{2}+\mu \boldsymbol{b}:\left[\boldsymbol{\varepsilon}^{\mathrm{e}} \cdot \boldsymbol{b} \cdot \boldsymbol{\varepsilon}^{\mathrm{e}}\right],
$$

where $\Lambda$ and $\mu$ are the Lamé parameters. The evolving anisotropic damage is modeled by the integrity tensor $\boldsymbol{b}$ of second order. $\boldsymbol{b}=\boldsymbol{I}$ denotes an undamaged state while $\boldsymbol{b}=\mathbf{0}$ denotes a fully damaged state.

Following [12], the thermodynamic forces being dual to $(\boldsymbol{\varepsilon}, \boldsymbol{b})$ follow from Eq. (5) in a straightforward manner as

$$
\boldsymbol{\sigma}=\frac{\partial \Psi}{\partial \boldsymbol{\varepsilon}}=\Lambda \operatorname{tr}\left(\boldsymbol{b} \cdot \boldsymbol{\varepsilon}^{\mathrm{e}}\right) \boldsymbol{b}+2 \mu \boldsymbol{b} \cdot \boldsymbol{\varepsilon}^{\mathrm{e}} \cdot \boldsymbol{b}, \quad \boldsymbol{\beta}=-\frac{\partial \Psi}{\partial \boldsymbol{b}}=-\Lambda \operatorname{tr}\left(\boldsymbol{b} \cdot \boldsymbol{\varepsilon}^{\mathrm{e}}\right) \boldsymbol{\varepsilon}^{\mathrm{e}}-2 \mu \boldsymbol{\varepsilon}^{\mathrm{e}} \cdot \boldsymbol{b} \cdot \boldsymbol{\varepsilon}^{\mathrm{e}}
$$

where $\boldsymbol{\beta}$ can be interpreted as a tensor-valued energy release rate.

The constitutive model is completed in terms of Generalized Standard Materials [13]. Following [13] the evolution equations are defined as gradients of a plastic potential $g$, i.e.,

$$
\dot{\boldsymbol{\varepsilon}}^{\mathrm{p}}=\lambda \partial_{\sigma} g, \quad \dot{\boldsymbol{b}}=\lambda \partial_{\beta} g \quad \text { with } \quad g=\Phi(\boldsymbol{\sigma} ; \boldsymbol{b})+\Gamma_{\beta}(\boldsymbol{\beta} ; \boldsymbol{b}),
$$

where $\lambda \geq 0$ is the plastic multiplier. Choosing a von Mises type function, yield function

$$
\Phi=\sqrt{\sigma_{\mathrm{eq}}}-\sigma_{y} \quad \text { with } \quad \sigma_{\mathrm{eq}}=\frac{3}{2}\left[\operatorname{tr}\left(\left[\boldsymbol{b}^{-1} \cdot \boldsymbol{\sigma}\right]^{2}\right)-\frac{1}{3} \operatorname{tr}\left(\boldsymbol{b}^{-1} \cdot \boldsymbol{\sigma}\right)^{2}\right]
$$

is adopted, where $\sigma_{\text {eq }}$ is a generalized equivalent von Mises stress measure. Finally potential $\Gamma_{\beta}$ is defined as

$$
\Gamma_{\boldsymbol{\beta}}=\frac{\eta_{1}}{2} \operatorname{tr}(\boldsymbol{b} \cdot \boldsymbol{\beta})^{2}+\frac{\eta_{2}}{2} \boldsymbol{b}:[\boldsymbol{\beta} \cdot \boldsymbol{b} \cdot \boldsymbol{\beta}],
$$

where the model parameter $\eta_{1}$ defines the isotropic part of the damage evolution and $\eta_{2}$, in turn, the anisotropic part.

\section{Micromorphic regularization}

As stated above, the local constitutive model is ill-posed from a mathematical point of view. This ill-posedness results in mesh dependent results as far as the finite element method is concerned. In order to bypass this ill-posedness, the local model will be enhanced in line with the micromorphic approach, see $[3,4]$. 


\subsection{Standard approach}

The softening behavior of the anisotropic damage model introduced in Section 3 is determined by the monotonically decreasing second-order tensor $\boldsymbol{b}$ (its eigenvalues decrease and converge towards zero). In order to regularize the local constitutive model, the gradient of $\boldsymbol{b}$ shall be incorporated into the model. Following [3, 4], the regularization is not directly applied to $b$, but to an additional second-order tensor $\varphi_{b}$. With this tensor, a micromorphic approximation of a standard gradient regularization is obtained by means of enhanced potential

$$
\Pi^{\mathrm{enh}}=\int_{\mathcal{B}} \Psi^{\mathrm{enh}} \mathrm{d} V=\int_{\mathcal{B}}\left[\Psi+\Psi^{\mathrm{pen}}+\Psi^{\mathrm{grad}}\right] \mathrm{d} V, \quad \Psi^{\text {pen }}=\frac{c_{\boldsymbol{b}}}{2}\left\|\varphi_{\boldsymbol{b}}-\boldsymbol{b}\right\|^{2}, \quad \Psi^{\mathrm{grad}}=\frac{l_{\boldsymbol{b}}}{2}\left\|\nabla \varphi_{\boldsymbol{b}}\right\|^{2} .
$$

Here, $\Psi$ denotes the Helmholtz energy of the unregularized underlying local damage model presented in the previous section and $l_{b}$ is a length parameter which defines the zone showing localized material damage. If energy minimization governs the evolution of field $\varphi_{b}$ and if, furthermore, penalty parameter $c_{b}$ is chosen as sufficiently large, $\varphi_{b}$ converges towards $b$ and both spatial distributions coincide.

Assuming energy minimization as the overriding principle the gradient regularization results from stationary of potential (10). To be more explicit,

$$
\delta_{\varphi_{b}} \Pi^{\mathrm{enh}}=\int_{\mathcal{B}} c_{b}\left[\varphi_{b}-b\right]: \delta \varphi_{b}+l_{b} \nabla \varphi_{b} \vdots \nabla \delta \varphi_{b} \mathrm{~d} V,
$$

which can be transformed into Laplace type

$$
l_{b} \operatorname{div} \nabla \varphi_{b}=c_{b}\left[\varphi_{b}-b\right]
$$

Its insertion into $\beta^{\text {enh }}$ leads to

$$
\beta^{\text {enh }}=-\frac{\partial \Psi^{\text {enh }}}{\partial \boldsymbol{b}}=\underbrace{-\frac{\partial \Psi}{\partial \boldsymbol{b}}}_{=\boldsymbol{\beta}}-\frac{\partial \Psi^{\text {pen }}}{\partial \boldsymbol{b}}=\underbrace{\beta}_{\text {local model }}+l_{\boldsymbol{b}} \operatorname{div} \nabla \varphi_{\boldsymbol{b}}
$$

and, hence, the gradient extension becomes obvious.

Although the model is indeed gradient-enhanced, it does - surprisingly — not regularize the local damage model.

\subsection{Novel Approach}

As it is seen in Section 6.1 the standard approach fails in regularizing the anisotropic ductile damage model. This is due to the one-directional coupling between integrity tensor $\boldsymbol{b}$ and its global counterpart $\varphi_{b}$, i.e., an evolution of $b$ leads to an evolution of $\varphi_{b}$, but not vice versa. In order to obtain a two-directional coupling, tensor

$$
\omega_{b}=\frac{\partial \Psi^{\text {enh }}}{\partial \varphi_{b}}=c_{b}\left[\varphi_{b}-b\right] \stackrel{\text { Eq. (12) }}{=} l_{b} \operatorname{div} \nabla \varphi_{b}
$$

being dual to $\varphi_{b}$ is introduced. Evidently, this variable includes a gradient contribution. Furthermore, $\omega_{b}$ connects the local energy release rate tensor $\beta$ and its gradient-enhanced counterpart $\boldsymbol{\beta}^{\text {enh }}$ according to

$$
\beta^{\mathrm{enh}}=\beta+\omega_{b} .
$$


Based on Eq. (15), the yield function is modified according to

$$
\Phi^{\mathrm{enh}}=\Phi+f_{\omega_{b}} \quad \text { with } \quad f_{\omega_{b}}\left(\omega_{b}\right)=-\sqrt{\boldsymbol{b}^{-1}}: \omega_{\boldsymbol{b}} .
$$

Clearly, choice (16) is not unique and can be interpreted as a gradient dependent yield limit, i.e., $\sigma_{y}^{\text {enh }}=\sigma_{y}-f_{\omega_{b}}$. Furthermore, the yield function of the original local model and that of the modified counterpart according to Eq. (16) are only different as long as $f_{\omega_{b}} \neq 0$, i.e., $b \neq \varphi_{b}$. In order to keep the plastic potential of the original local model, it is replaced by (compare to Eq. (9))

$$
\begin{aligned}
\Gamma_{\boldsymbol{\beta}}^{\mathrm{enh}} & =\frac{\eta_{1}}{2} \operatorname{tr}\left(\boldsymbol{b} \cdot\left[\boldsymbol{\beta}^{\mathrm{enh}}-\omega_{\boldsymbol{b}}\right]\right)^{2}+\frac{\eta_{2}}{2} \boldsymbol{b}:\left[\left[\boldsymbol{\beta}^{\mathrm{enh}}-\omega_{\boldsymbol{b}}\right] \cdot \boldsymbol{b} \cdot\left[\boldsymbol{\beta}^{\mathrm{enh}}-\omega_{\boldsymbol{b}}\right]\right] \\
& =\frac{\eta_{1}}{2} \operatorname{tr}(\boldsymbol{b} \cdot \boldsymbol{\beta})^{2}+\frac{\eta_{2}}{2} \boldsymbol{b}:[\boldsymbol{\beta} \cdot \boldsymbol{b} \cdot \boldsymbol{\beta}] .
\end{aligned}
$$

\section{Regularized isotropic ductile damage - Constitutive Framework}

Material degradation like nucleation of pores and growth of micro cracks are anisotropic in nature. However, an isotropic description of these defects, which is computationally significantly more efficient, is often sufficient, e.g., if only proportional loading paths occur. Such an isotropic approximation of the anisotropic damage model presented before is given here.

In order to simplify the anisotropic damage model, the integrity tensor is assumed to be of spherical structure, i.e.,

$$
\boldsymbol{b}=b \boldsymbol{I} .
$$

With assumption (18) the resulting Helmholtz energy of the isotropic damage model reads

$$
\Psi=\frac{\Lambda}{2} b^{2} \operatorname{tr}\left(\varepsilon^{\mathrm{e}}\right)^{2}+\mu b^{2} \varepsilon^{\mathrm{e}}: \boldsymbol{\varepsilon}^{\mathrm{e}} .
$$

Analogously to potential (10), the micromorphic enhanced potential for an isotropic material degradation follows as

$$
\Pi^{\mathrm{enh}}=\int_{\mathcal{B}} \Psi^{\mathrm{enh}} \mathrm{d} V=\int_{\mathcal{B}}\left[\Psi+\Psi^{\mathrm{pen}}+\Psi^{\mathrm{grad}}\right] \mathrm{d} V, \quad \Psi^{\mathrm{pen}}=\frac{c_{b}}{2}\left[\varphi_{b}-b\right]^{2}, \quad \Psi^{\mathrm{grad}}=\frac{l_{b}}{2}\left\|\nabla \varphi_{b}\right\|^{2}
$$

and hence, the thermodynamic forces take the form

$$
\begin{aligned}
\sigma & =\frac{\partial \Psi^{\mathrm{enh}}}{\partial \boldsymbol{\varepsilon}}=\Lambda b^{2} \operatorname{tr}\left(\boldsymbol{\varepsilon}^{\mathrm{e}}\right) \boldsymbol{I}+2 \mu b^{2} \varepsilon^{\mathrm{e}}, \quad \omega_{b}=\frac{\partial \Psi^{\mathrm{enh}}}{\partial \varphi_{b}}=c_{b}\left[\varphi_{b}-b\right]=l_{b} \operatorname{div} \nabla \varphi_{b}, \\
\beta^{\mathrm{enh}} & =-\frac{\partial \Psi^{\mathrm{enh}}}{\partial b}=-\Lambda b \operatorname{tr}\left(\boldsymbol{\varepsilon}^{\mathrm{e}}\right)^{2}-2 b \mu \varepsilon^{\mathrm{e}}: \varepsilon^{\mathrm{e}}+c_{b}\left[\varphi_{b}-b\right]=\beta+\omega_{b} .
\end{aligned}
$$

Likewise, assumption (18) and micromorphic modifications (16) and (17) affect the plastic potential. To be more explicit,

$$
\begin{aligned}
\Phi^{\mathrm{enh}} & =\sqrt{\sigma_{\mathrm{eq}}}-\tau_{y}+f_{\omega_{b}} \quad \text { with } \quad \sigma_{\mathrm{eq}}=\frac{3}{2} b^{-2} \sigma_{\mathrm{dev}}: \sigma_{\mathrm{dev}}, \quad f_{\omega_{b}}=-\sqrt{b^{-1}} \omega \\
\Gamma_{\beta} & =\frac{\eta}{2} b^{2}\left[\beta^{\mathrm{enh}}-\omega_{b}\right]^{2}=\frac{\eta}{2} b^{2} \beta^{2},
\end{aligned}
$$

where the modifications result again in a two-directional coupling between local integrity variable $b$ and global counterpart $\varphi_{b}$. 


\section{Numerical Examples}

This section visualizes the mechanical features of the novel regularization technique. First, the general features of the model are shown on the example a one-dimensional truss with an imperfection. Afterwards, the example of a plate with a centered hole is investigated.

\subsection{Imperfect element test - 1D}

The truss is fixed on the left hand side and is loaded in tension by prescribing the displacement at the right hand side. The mechanical setup, the finite element discretization by means of $(20,40,80$ and 160) linear displacement-driven finite elements, together with the model parameters, are summarized in Fig. 2. In order to trigger localization, the element on the right hand side is weakened by reducing its initial yield stress by $5 \%$.

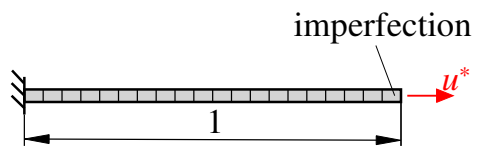

\begin{tabular}{ccccc}
\multicolumn{5}{c}{ Material parameter } \\
\hline$E$ & $\sigma_{y}$ & $\eta$ & $c_{b}$ & $l_{b}$ \\
400 & $1(0.95)$ & 2000 & 5 & 100 \\
\hline
\end{tabular}

Figure 2. One-dimensional truss with imperfection: Description of the boundary value problem and model parameters

The mechanical responses as predicted by the three different models are summarized in Fig. 3. Accordingly, the underlying local model as well as its modified micromorphic exten-
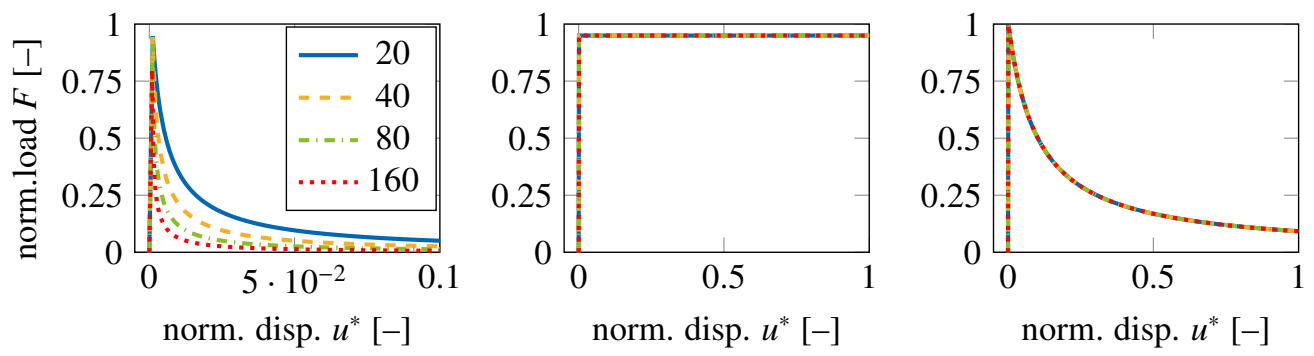

Figure 3. One-dimensional truss with imperfection: Load-displacement diagrams predicted by: (left) the local model (different scale), (middle) the standard micromporphic model and (right) novel micromorpic model for different finite element meshes (20, 40, 80 and 160 finite elements)

sion capture the desired softening response. By way of contrast, the standard straightforward micromorphic model does not, but shows a plateau in the force-displacement diagram. Without going too much into details, the naive micromorphic extension eliminates the evolution of $b$ within the localized element such that the total mechanical response is not characterized by material softening anymore.

For the sake of completeness, mesh objectivity of the novel model is shown in Fig. 3 (right). While the load-displacement diagrams associated with the local model depend on the underlying finite element mesh, this pathological behavior is neither observed for the standard micromorphic nor for the novel micromorphic model. However and as already mentioned before, only the novel micromorphic model captures the desired softening response. 


\subsection{Plate with a centered hole}

In this subsection the example of a plate with a centered hole is investigated. Furthermore, the anisotropic damage model is extended by nonlinear isotropic and kinematic hardening (corresponding to model parameters $H, B$ and $z$ ) according to $[14,15]$. The mechanical setup and the material parameters are presented in Fig. 4. Due to symmetry only the blue framed area is calculated and provided with boundary conditions preserving the symmetry of the system.

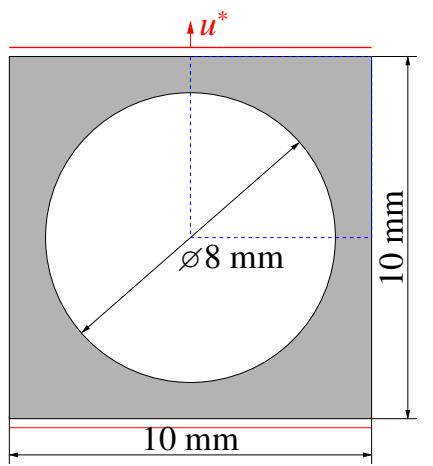

Material parameter

\begin{tabular}{cc|c||cc|c}
\hline$E$ & {$[\mathrm{MPa}]$} & 400 & $\eta_{1}$ & $\frac{1}{\mathrm{MPa}}$ & 500 \\
$v$ & {$[-]$} & 0.3 & $\eta_{2}$ & $\frac{1}{\mathrm{MPa}}$ & 500 \\
$\tau_{y}$ & {$[\mathrm{MPa}]$} & 1 & $z$ & {$[-]$} & 0.5 \\
$H$ & {$[\mathrm{MPa}]$} & 100 & $c_{\boldsymbol{b}}$ & {$[\mathrm{MPa}]$} & 10 \\
$B$ & {$[\mathrm{MPa}]$} & 5 & $l_{\boldsymbol{b}}$ & {$[\mathrm{N}]$} & 1 \\
\hline
\end{tabular}

Figure 4. Two-dimensional plate with a centered hole: Description of the boundary value problem and model parameters (including parameters for isotropic and kinematic hardening)

The structural response in terms of load-displacement-diagrams and distributions of the integrity tensor component $b_{y y}$, loading direction, is visualized in Fig. 5 and 6 for three different finite element triangulations (137, 461 and 991 elements). According to Fig. 5(left) the
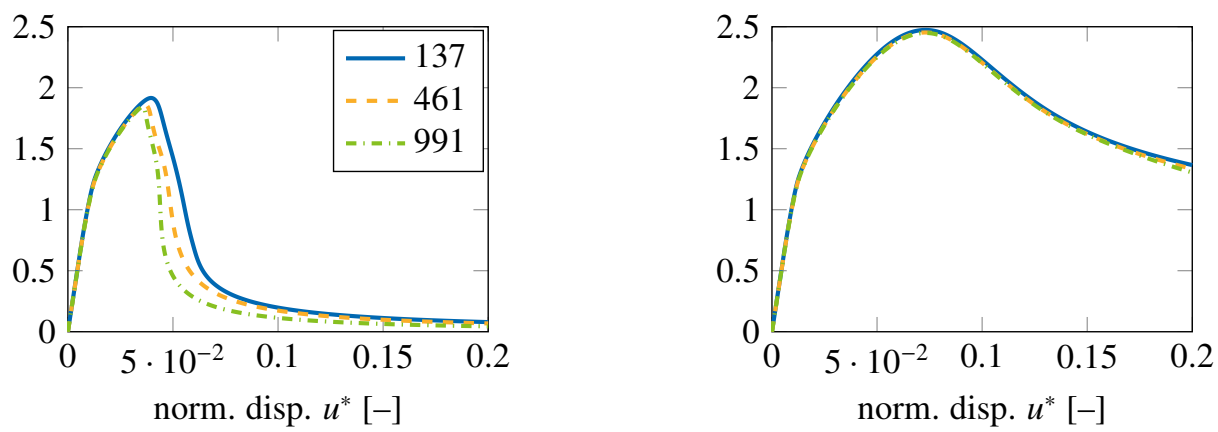

Figure 5. Two-dimensional plate with a centered hole: Load-displacement diagrams predicted by: (left) the local modal and (right) the novel micromorphic model for different finite element meshes (137, 461, 991 elements)

local model shows pathological mesh dependence. By way of contrast, the novel regularized model, Fig. 5(right), does not. The distribution of integrity tensor component $b_{y y}$ is shown in Fig. 6 for the final displacement amplitude of $0.2 \mathrm{~mm}$. Again, the three distributions agree reasonably well. Hence, mesh objectivity is confirmed. 


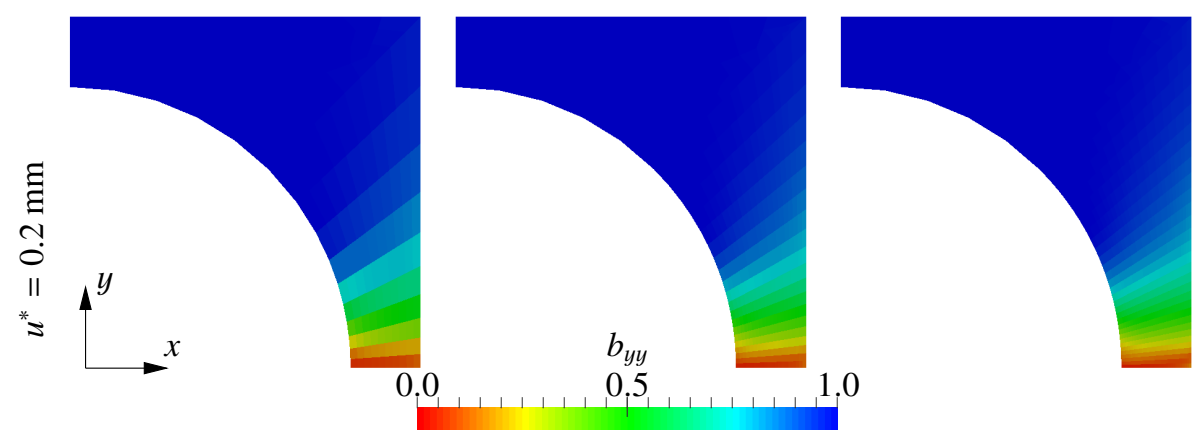

Figure 6. Two-dimensional plate with a centered hole: Distributions of component $b_{y y}$ of integrity tensor $b$ predicted by the novel micromorphic model for three different finite element meshes $(137,461$, 991 elements)

Financial support from the German Research Foundation (DFG) via SFB/TR TRR 188 (projectnumber 278868966), project C01, is gratefully acknowledged.

\section{References}

[1] A.-M. Abellan, R. de Borst, Computer Methods in Applied Mechanics and Engineering 195 (37), 5011-5019 (2005)

[2] R. H. J. Peerlings, M. G. D. Geers, R. de Borst, W. A. M. Brekelmans, International Journal of Solids and Structures 38 (44), 7723-7746 (2001)

[3] S. Forest, International Journal of Engineering Mechanics 135 (3), 117-131 (2009)

[4] B. J. Dimitrijevic, K. Hackl, International Journal for Numerical Methods in Biomedical Engineering 27 (8), 1199-1210 (2011)

[5] L. M. Kachanov, Otdelenie Teckhnicheskikh Nauk, Izvestiia Akademii Nauk SSSR 8, 2631 (1958)

[6] J. Lemaitre, Journal of Engineering Materials and Technology 107 (1), 83-89 (1985)

[7] A. Menzel, M. Ekh, P. Steinmann, K. Runesson, International Journal for Numerical Methods in Engineering 54 (10), 1409-1430 (2002)

[8] M. Brünig, V. Hagenbrock, S. Gerke, ZAMM - Journal of Applied Mathematics and Mechanics / Zeitschrift für Angewandte Mathematik und Mechanik 98 (2), 181-194 (2018)

[9] P. Steinmann, I. Carol, International Journal of Engineering Science 36 (15), 1793-1814 (1998)

[10] M. Brünig, S. Ricci, International Journal of Plasticity 21 (7), 1346-1382 (2005)

[11] R. K. Abu Al-Rub, G. Z. Voyiadjis, International Journal of Damage Mechanics 18 (2), 115-154 (2009) .

[12] B. D. Coleman and W. Noll, Journal of Chemical Physics 47 (2), 597-613 (1967)

[13] B. Halphen, Q. S. Nguyen, Journal de Mécanique 14 (1), 39-63 (1975)

[14] M. Ekh, A. Menzel, K. Runesson, P. Steinmann, International Journal of Engineering Science 41 (13-14), 1535-1551 (2003)

[15] C. O. Frederick, P. J. Armstrong, Materials at High Temperatures 24 (1), 1-26 (2007) 